\title{
CORRIGENDUM
}

\section{Preferential sensitivity of hematopoietic (HPs) and mesenchymal (MPs) progenitors to fludarabine suggests impaired bone marrow niche and HP mobilization}

MG Berger ${ }^{1,2,3}$, J Berger
V Maguer-Satta
$4,5,6,7$

${ }^{1}$ Hematology (Biology), CHU Hôtel-Dieu, Clermont-Ferrand, France; ${ }^{2}$ UPRES EA 3846, Faculté Médecine, Université de Clermont 1, Clermont-Ferrand, France; ${ }^{3}$ Department of Hematology and Cell Therapy, CHU Hôtel-Dieu, Clermont-Ferrand, France; ${ }^{4}$ Université de Lyon, Lyon, France; ${ }^{5}$ ISPB, Université de Lyon 1, Lyon, France; ${ }^{6}$ INSERM, U590, Lyon, France; ${ }^{7}$ IFR62, Lyon, France and ${ }^{8}$ Department of Hematology, E Herriot Hospital, Lyon, France

Leukemia (2008) 22, 2135; doi:10.1038/leu.2008.202

Correction to: Leukemia advance online publication, 3 July 2008; doi:10.1038/leu.2008.167

Since the publication of the above paper, the authors have noticed errors in Dr $\vee$ Maguer-Satta's name and also in the affiliations. The correct name and affiliations are shown above.

The authors apologize for any inconvenience caused. 